\title{
Class-E Amplifier and Rectifier for a Wireless Link with Secure Signal and Simultaneous Power Transmission
}

\author{
Leysi Rizo ${ }^{1}$, David Vegas ${ }^{1}$, M. Nieves Ruiz ${ }^{1}$, R. Marante ${ }^{2}$, L. Cabria ${ }^{2}$ and José A. García ${ }^{1}$ \\ ${ }^{1}$ Dpto. Ing. Comunicaciones, Universidad de Cantabria, Plaza de la Ciencia s/n, 39005 Santander, Spain. \\ ${ }^{2}$ TTI Norte, c/Albert Einstein n ${ }^{\circ}$ 14, 39011, Santander, Spain \\ joseangel.garcia@unican.es
}

\begin{abstract}
In this paper, the design and implementation of a GaN HEMT class-E power amplifier and a Schottky diode class$E$ rectifier are described. Based on the widely known Linear Amplification with Nonlinear Components technique (LINC), and taking advantage of the spatial power combining capability of a dual-fed square patch, the highly efficient amplifier and rectifier may be incorporated at both sides of a wireless link, proposed for the simultaneous transmission of signal and power to a remote receiver.

Index Terms - Class-E, GaN HEMT, LINC, power amplifier, rectifier, wireless power transmission.
\end{abstract}

\section{INTRODUCTION}

Wireless power [1] and highly efficient signal transmission [2] are probably two of the topics receiving more attention from the microwave community. The increased use of switched-mode power amplifiers (SMPAs) for the transmission of spectrally efficient signals, with an associated high peak-to-average power ratio (PAPR), coincides in time with the introduction of their time-reverse duals [3] for $\mathrm{RF} /$ microwave-to-DC conversion.

The Linear Amplification with Nonlinear Components, proposed by Cox in [4] stands as one of the few techniques allowing the linear reproduction of a strongly-varying envelope signal using SMPAs. Incorporating an isolated hybrid combiner, instead of the reactive counterpart proposed by Chrieix in his seminal work [5], a relatively simple correction of the amplitude and phase imbalance is possible at the expense of a poor average efficiency. Being that high values of the outphasing angle may be quite frequent when handling signals with demanding envelope dynamics, the significant power lost in the isolated port resistor could be either reduced through more complex multilevel approaches [6], or turned back into DC for being recycled [7] (Fig. 1).

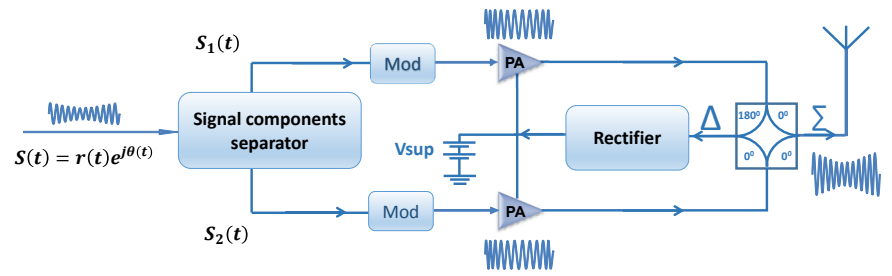

Fig. 1. LINC architecture with the power reuse concept [7].

In this paper, a wireless signal and power transmission (WSPT) link architecture is proposed, translating the power reuse concept of [7] to a far-field placed receiver. The design and implementation of a GaN HEMT class-E amplifier and a Schottky diode class-E rectifier are then described for their use as high efficiency parts in the transmitting and receiving sides, respectively. A single-radiator implementation is added for validation.

\section{Proposed SySTEM OvERVIEW}

In Fig. 2, a simplified system diagram of the proposed WSPT link is presented. A two-tone excitation, traditional for intermodulation distortion (IMD) experiments, has been used for illustration purposes. After being amplified by class-E SMPAs, the constant envelope phase-modulated (PM) signals, bearing an additional $90^{\circ}$ phase difference, are used for exciting the orthogonal $\mathrm{TM}_{01}$ and $\mathrm{TM}_{10}$ modes of a $4 \times 1$ array of dual-fed aperture-coupled square patches in the $915 \mathrm{MHz}$ band. Taking advantage, as in [8], of its spatial power combining capabilities, the desired communication signal, usually obtained from the $\Sigma$ port of a $180^{\circ}$ hybrid, could be transmitted in this case with a right-handed circular polarization (RHCP). At the same time, the undesired component to be recycled, traditionally derived from the $\Delta$ port of the isolated combiner, would be also radiated with the orthogonal left-handed circular polarization (LHCP).

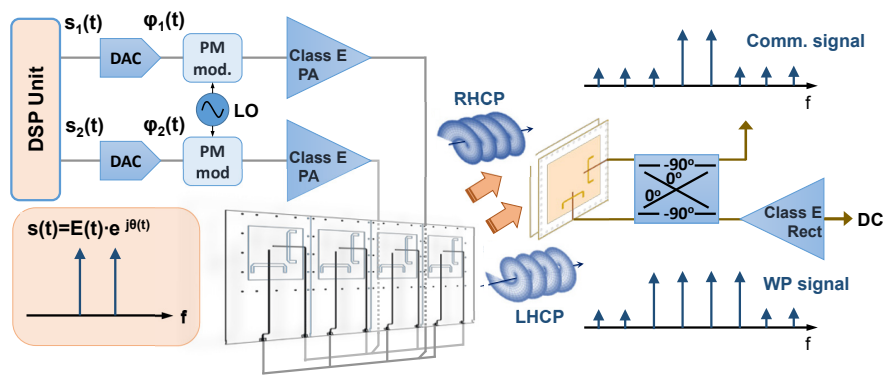

Fig. 2. Simplified diagram of the proposed WSPT architecture.

In the remote side, both components could be easily separated thanks to the connection of the vertical and horizontal outputs of a dual-fed square patch to a quadrature hybrid coupler. While the information in the desired RHCP signal could be recovered by the receiver, a class-E rectifier would allow turning the power of the undesired transmitted component into the required DC for biasing it. This link would also add security layers in polarization and azimuth. The reception of the transmitted signal, either from other directions 
with a RHCP antenna, or even at broadside if employing a linearly polarized one, could be prevented.

\section{GAN HEMT CLASS-E PA}

For the class-E power amplifying branches of the LINCbased transmitting array, a packaged CGH35030F GaN HEMT on SiC substrate from Cree Inc., was selected. From the value of the characterized equivalent output capacitance at $V_{D S}=28 \mathrm{~V}$ and $V_{G S}=-3.7 \mathrm{~V}\left(C_{\text {out }}=3.6 \mathrm{pF}\right)$, the nominal complex impedance at the fundamental was estimated to be $Z_{d}(f)=(0.1836+\mathrm{j} \cdot 0.2116) /\left(\omega \cdot C_{\text {out }}\right)=8.9+\mathrm{j} \cdot 10.2 \Omega$ from [9]. This departing value and the open circuit conditions at the second- and third-harmonic were then synthesized through a lumped element multi-resonant drain terminating network derived from [10]. At gate side, a capacitor to ground and a small length of microstrip line were enough for input matching. In Fig. 3, the schematic and photograph of one of the implemented class-E PAs are shown.

a)

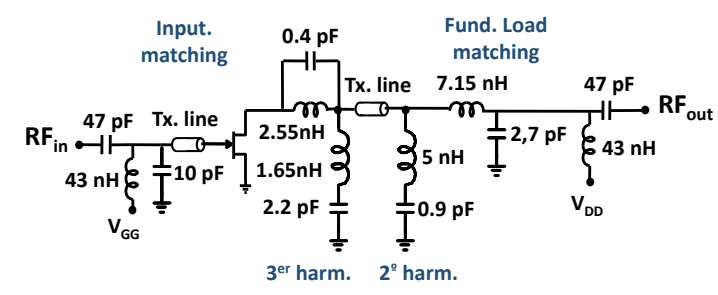

b)

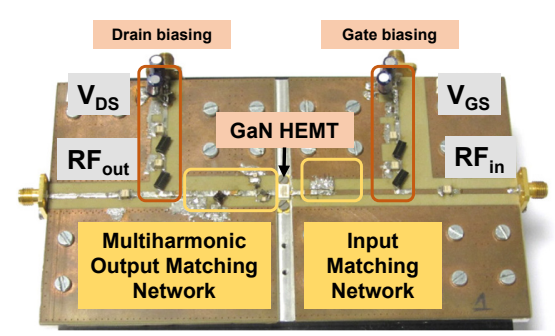

Fig. 3. Designed class-E PA: a) circuit schematic, and b) photograph. Micro/Mini Air Core inductors from Coilcraft were used, together with 100A/100B capacitors from ATC.

The measured evolution with frequency for the output power and the efficiency may be appreciated in Fig. 4. The drain efficiency stays over $80 \%$ along a $110 \mathrm{MHz}$ range. No significant differences in terms of output power and efficiency were found between the two implemented SMPAs.

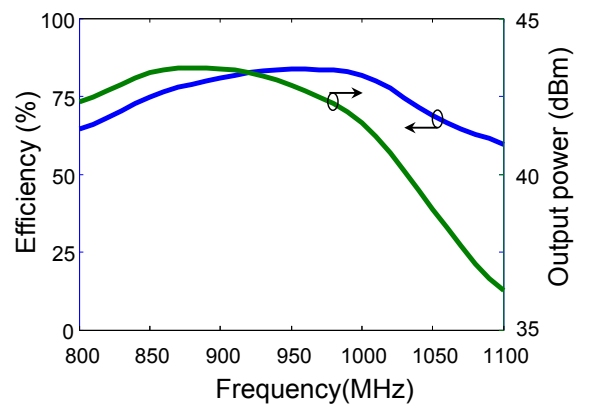

Fig. 4. Output power and efficiency evolution with frequency.

\section{SCHOTTKY Diode ClASS-E ReCTIFIER}

For the class-E rectifier in the receiving side of the link, an HSMS-282 Schottky diode from Avago Technologies was employed. With the measured value of its OFF-state capacitance, $C_{j}=0.76 \mathrm{pF}$, the nominal impedance to be synthesized at the fundamental was estimated to be $Z_{d}(f)=42$ $+\mathrm{j} \cdot 48.4 \Omega$. For simplicity and compactness, the poly-harmonic terminating network was here based on a $25 \mathrm{nH}$ ultraminiature Air Core inductor, also from Coilcraft, with a parasitic resonance at $2.5 \mathrm{GHz}$ (between the second- and thirdharmonic). This inductor was resonated slightly below 915 $\mathrm{MHz}$ thanks to a series $4.7 \mathrm{pF} 100 \mathrm{~A}$ ATC capacitor. A parallel tank was used, instead of the theoretical choke inductor, in order to force a high reactance with a small-valued and sized high Q coil. The rectifier schematic and a photograph with implementation details are shown in Fig. 5.

a)

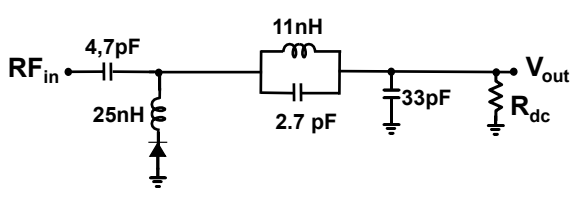

b)

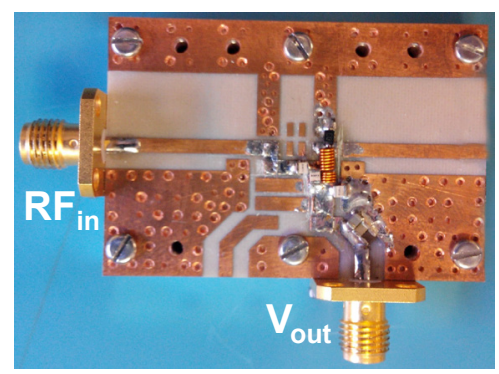

Fig. 5. Designed class-E rectifier a) Circuit schematic, and b) photograph with implementation details.

The measured output voltage and efficiency over a $214 \Omega$ load (the optimum performance appeared for lighter loading conditions than the one predicted by class-E theory, due in part to the non-negligible diode parasitic resistance) are plotted in Fig. 6a as a function of frequency, while in Fig. 6b in terms of the input power. A peak efficiency value of $74 \%$ was estimated for an input power of $23 \mathrm{dBm}$.

a)

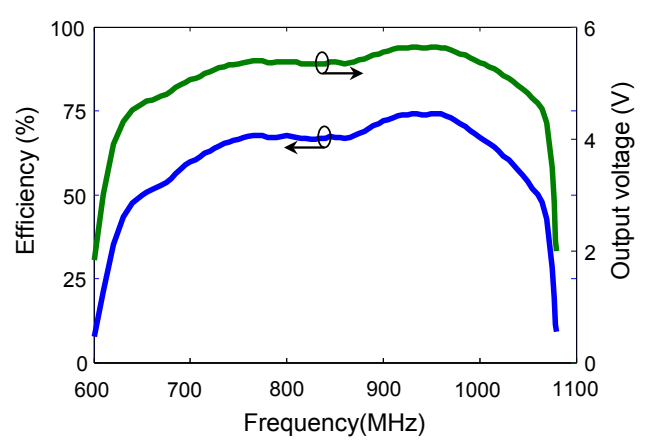


b)

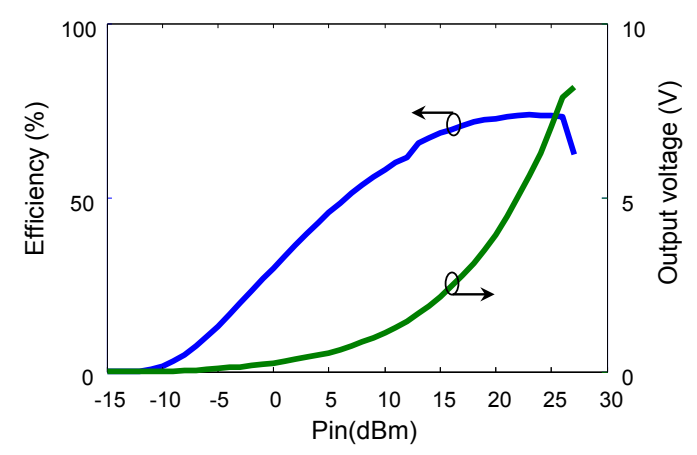

Fig. 6. Output voltage and efficiency versus a) frequency and b) input power, as measured for the implemented class-E rectifier.

\section{SingLe-ELEMENT VALIDATION}

In order to validate the proposed approach in a simple way, while also testing the designed SMPAs, a single-element version of the architecture in Fig. 2 was implemented. A pair of tones with $100 \mathrm{kHz}$ frequency spacing was selected for the experiment. Vector signal generation and analysis capabilities were also incorporated into a far-field test set-up (see Fig. 7).

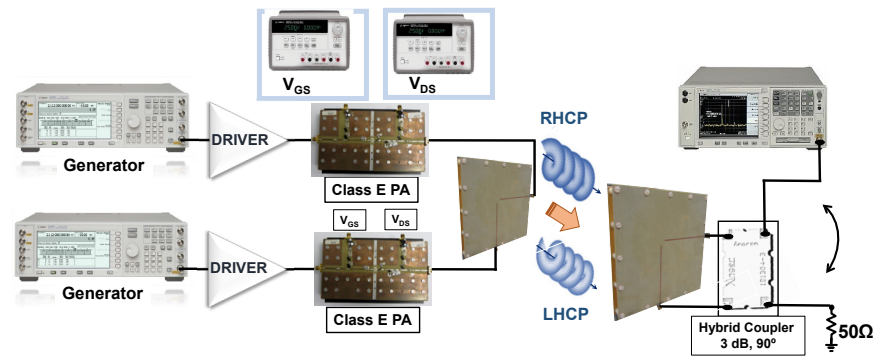

Fig. 7. Simplified diagram of the employed far-field set-up.

The phase modulating signals, with the envelope of the two-tone excitation (following a $\left.\left|\cos \left[1 / 2 \cdot\left(\omega_{2}-\omega_{1}\right) \cdot t\right]\right|\right)$ coded into the outphasing angle, were sent to the generators. Their relative time delay, phase and power level were carefully adjusted to correct the existing imbalances. With the aid of the VSA, the recovered signals were then analyzed.
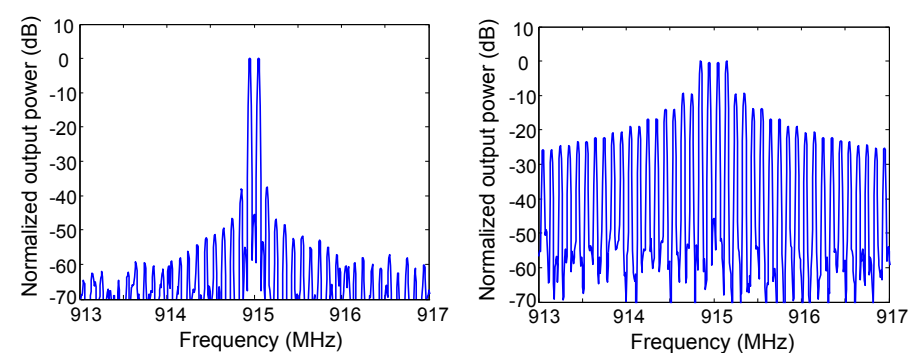

a)

b)

Fig. 8. Measured spectrum at the output ports of the $90^{\circ}$ hybrid: a) received signal and $b$ ) powering component.

As observed from Fig. 8a, the desired two tones were obtained from one of the output ports of the $90^{\circ}$ hybrid (an off-the-shelf part from Anaren). Low-level IMD products may be appreciated, together with a small component at the carrier position (associated to non-idealities in the IQ modulators of the employed generators). The measured spectrum in the remaining output port, where the previously designed rectifier would be in charge of DC powering the receiving circuitry, is also presented in Fig. 8b. It approximately corresponds, as expected, to a signal with similar envelope and average power, but with the $180^{\circ}$ phase transitions coinciding in time with the amplitude maxima instead of the nulls.

\section{CONCLUSION}

The design and implementation of a GaN HEMT class-E power amplifier and a Schottky diode class-E rectifier, aimed to be used as efficient power transducers at both sides of a WSPT link, have been presented in this paper. The proposed architecture, based on the LINC technique, has been validated for the classical discrete-spectrum two-tone excitation, using a single radiating element for transmission.

\section{ACKNOWLEDGEMENT}

This work has been supported by the Spanish Ministry of Economy and Competitiveness (MINECO) through the TEC2014-58341-C4-1-R project with FEDER co-funding. David Vegas also thanks the support provided by the predoctoral grant BES-2015-072203.

\section{REFERENCES}

[1] N. B. Carvalho et. al., "Wireless Power Transmission: R\&D Activities Within Europe," IEEE Trans. Microwave Theory \& Tech., vol. 62, no. 4, pp. 1031-1045, April 2014.

[2] P. Lavrador, T.R. Cunha, P. Cabral and J. C. Pedro, "The Linearity-Efficiency Compromise," in IEEE Microwave Mag., vol. 11 , no.5, pp. 44-58, Aug. 2010

[3] D. C. Hamill, "Time Reversal Duality and the Synthesis of a Double Class E DC-DC Converter," 21st Power Electron. Specialist Conf., PESC'90, pp. 512-521, 1990.

[4] D. C. Cox, "Linear Amplification with Nonlinear Components," IEEE Trans. Comm., vol. COM-23, pp. 1942-1945, Dec. 1974.

[5] H. Chireix, "High Power Outphasing Modulation," Proc. IRE, vol. 23, no. 11, pp. 1370-1392, Nov. 1935.

[6] C. SungWon et. al., "Asymmetric Multilevel Outphasing Architecture for Multi-standard Transmitters," in 2009 IEEE RF Integrated Circuits Symp., pp.237-240, 7-9 June 2009.

[7] R. Langridge et. al., "A Power Re-use Technique for Improved Efficiency of Outphasing Microwave Power Amplifiers," IEEE Trans. Microw. Theory Tech., vol. 47, no. 8, pp. 1467-1470, Aug. 1999.

[8] S. Gao and P. Gardner, "Integrated Antenna/Power Combiner for LINC Radio Transmitters," in IEEE Trans. Microw. Theory Tech., vol. 53, no. 3, pp. 1083-1088, March 2005.

[9] R. Beltran and F. H. Raab, "Lumped-Element Output Networks for High-Efficiency Power Amplifiers, 2010 IEEE MTT-S Int. Microwave Symp., pp. 324-327, Anaheim, May 2010.

[10] F. H. Raab, "Idealized Operation of the Class E Tuned Power Amplifier," IEEE Trans. Circuits and Syst., vol. 24, no. 12, pp. 725- 735, Dec. 1977. 